IRA-International Journal of Technology \& Engineering

ISSN 2455-4480; Vol.04, Issue 01 (2016)

Institute of Research Advances

http://research-advances.org/index.php/IRAJTE

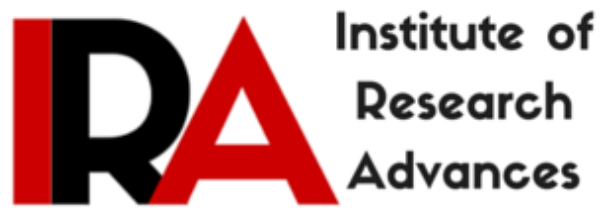

\title{
Optimality Analysis in K-Policy - Generalized Erlang Service Queueing Model
}

\author{
${ }^{1}$ S. Bhuvaneeswari, ${ }^{2}$ B. Ramesh Kumar, ${ }^{3}$ R.Solaiappan, ${ }^{4}$ S. Murugesan \\ ${ }^{1,2}$ Sree Sowdambika College of Engineering, Aruppukottai,Tamil Nadu, India. \\ ${ }^{3}$ Anna University Ramanathapuram Campus, Ramanathapuram, Tamil Nadu, India. \\ ${ }^{4}$ Sri.S.Ramasamy Naidu Memorial College, Sattur, TamilNadu, India.
}

DOI: http://dx.doi.org/10.21013/jte.v4.n1.p6

\section{How to cite this paper:}

Bhuvaneeswari, S., Kumar, B., Solaiappan, R., \& Murugesan, S. (2016). Optimality Analysis in K-Policy - Generalized Erlang Service Queueing Model. IRA-International Journal of Technology \& Engineering (ISSN 2455-4480), 4(1).

doi:http://dx.doi.org/10.21013/jte.v4.n1.p6

(C) Institute of Research Advances

(cc) EY-NC

This works is licensed under a Creative Commons Attribution-Non Commercial 4.0 International License subject to proper citation to the publication source of the work.

Disclaimer: The scholarly papers as reviewed and published by the Institute of Research Advances (IRA) are the views and opinions of their respective authors and are not the views or opinions of the IRA. The IRA disclaims of any harm or loss caused due to the published content to any party. 


\section{ABSTRACT}

This paper, we develops a nonlinear programming approach to construct the membership function of the performance measure in bulk arrival queueing system, in that the arrival and service rates are fuzzy numbers. The basic idea is transform to a "Generalized $k$-phase Erlang Fuzzy queue with infinite capacity to a family of conventional Generalized $k$-phase Erlang Service queue with infinite capacity by applying $\alpha$ - cut approach, and a pair of mixed integer nonlinear programs are formulated to calculate the upper and lower bound of the system performance measure at the different possibility level of $\alpha$. For practice use, the defuzzification of performance measures is also provided via Robust ranking method and carried out the defuzzification performance (Optimization) using Chi square distribution of the goodness fit and to analyze the interval of confidence optimality. A numerical example is included.

Key Words: Fuzzy Sets; Mixed integer nonlinear programming; k-phase Erlang Distribution; Reduced Chi-square Distribution; $\alpha$ - cut membership function.

\section{Introduction:}

In practical point of view the management would expect without blocking the arrival due to behavior of the service. If the system providing worst service then the level of queue size increased. Generally any management likes to avoid this kind of behaviour, in this connection we estimated the queues parameters used in statistical interference. The role of statistics (as contrasted with probability) in queueing analysis is focused on the estimation of arrival and service parameters and/or distribution from observed data. Since one must, in practice, often use observable data to decide on what arrival and service patterns of a queueing system actually are, it is extremely important to utilize the data to the fullest extent possible. Since there are many statistical problems associated with the simulation modeling in queueing analyses, the statistical procedures can help in making the best use of existing data, or in determining what and how much new data should be taken. That is an important fact of real queueing studies. The earliest work on the statistics of queues did, in fact, assume that the subject queue was fully observed over a period of time and therefore that complete information was available in the form of the arrival instants and the points of the beginning and each of the service of each customer. As would then be expected, the queue was assumed to be a Marko chain in process. Clarke (1957) began a sequence of papers on this and related topics by obtaining the maximum-likelihood estimators for the arrival and service parameters of an $\mathrm{M} / \mathrm{M} / \mathrm{I}$ queue, in addition to the variance -covariance matrix for the two statistics. This work was followed shortly thereafter by a similar exposition for $M / M / \infty$ by Benes (1957). In the queuing theory, it is usually assumed that the time between the two consecutive arrivals and the servicing time follow a special probability distribution. However, in the real world, this type of information is obtained using qualitative data and expressed by words like quick, medium, and slow rather than the probability distribution. It can be said that fuzzy queuing models are more realistic and practical than the classical ones. In this paper consider a queueing system in fuzzy environment to get a performace analysis. $[3,4,7,10,16,21]$ have alreay analyzed the fuzzy queueing theory and declared important results based on $\alpha$-level of membership function. Especially [2,11] are analyzed the queueing parameter via possibility theory used in fuzzy set theory and to determined the optimized the fuzzy numbers.[9] have analyze the fuzzy queueing system using parametric planning method. [5] has stated that the non-linear numerical mixed integer planning using possibility theory. Also the fuzzy ranking methods of Decooman [6], Liou and Wang [12] and Nakamura [13] are used for decision making in an uncertain environment. According to the researches done in this field, results obtained by Nakamura [13] are more precise than the other models therefore, in this paper, Nakamura method [22] has been used for fuzzy ranking. S. Barak[1] has proposed the two models of planning queuing systems and its effect on the cost of the each system by 
using two fuzzy queuing models of M/M/1 and M/E2/1. In the first section, we have compared two different fuzzy queuing models based on the costs of each model and fuzzy ranking methods are used to select optimal model due to the resulted complexity. [15]B.Palpandi proposed a procedure to find the various performance measures in terms of crisp values for bulk arrival queuing systems with varying fuzzy batch sizes where the arrival rate, service rate and batch sizes are fuzzy numbers. [17] W. Ritha et al., $\mathrm{N}$ policy queues with infinite capacity under uncertain arrival and service information. The purpose of this paper is to construct the membership function of the system characteristic of a N-policy queue with infinite capacity under imprecise data. [18] Satish Verma et al., analyzed the inter arrival and service pattens are using Chi-Square test method is being done to check the nature of arrival and service rate for the concerned bank of study by checking observed pattern with Null-Hypothesis, Poisson "es Distribution and Various other Patterns..The rest of the article follows as: in the second chapter, we deal with the Section2, the model under the steady state balance equation of Generalized Erlang K-phase service distribution. In Section 3, proposed a solution methodlogy. In section 4, we analyzie the MTNLP using the statistical interference. In Section 5, Numerical Example .

\section{Generalized Erlang K-phase service distribution:}

We now consider a model in which the service time has an Erlang type- $\mathrm{k}$ distribution. More specially, the overall service rate is assumed to be $\mu$, so that the rate of each service phase is $\mathrm{k} \mu$.Even though the service may not actually consist of $\mathrm{k}$ phases, Let $p_{n, i}(t)$ be the probability that in steady state there are " $n$ " customers in the system and the customer in service is in phase $\mathrm{i}(\mathrm{i}=1,2,3,4 \ldots . . \mathrm{k})$. Here, we number the phases backward, so $\mathrm{k}$ is the first phase of service and 1 is the last (a customer leaving phase 1 actually leaves the system). We can derive the steady state balance equation is :

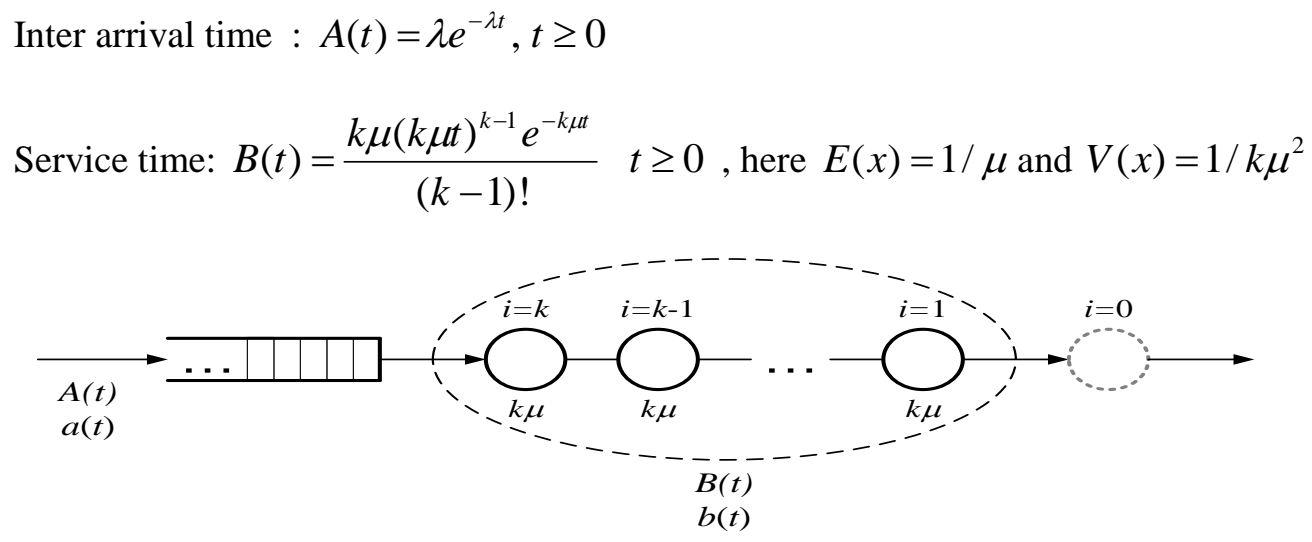

Define the 2-dim state variable (n,i) to be the total number of customers $n$ in the system and the customer being served is at $i$-stage (phase). Then $P(n)=\sum_{i=1}^{k} P(n, i)$

$$
\left(\begin{array}{l}
i=k: \text { at the } 1 \text { st phase } \\
i=1: \text { at the last phase } \\
i=0: \text { leaving the system or service completion }
\end{array}\right)
$$




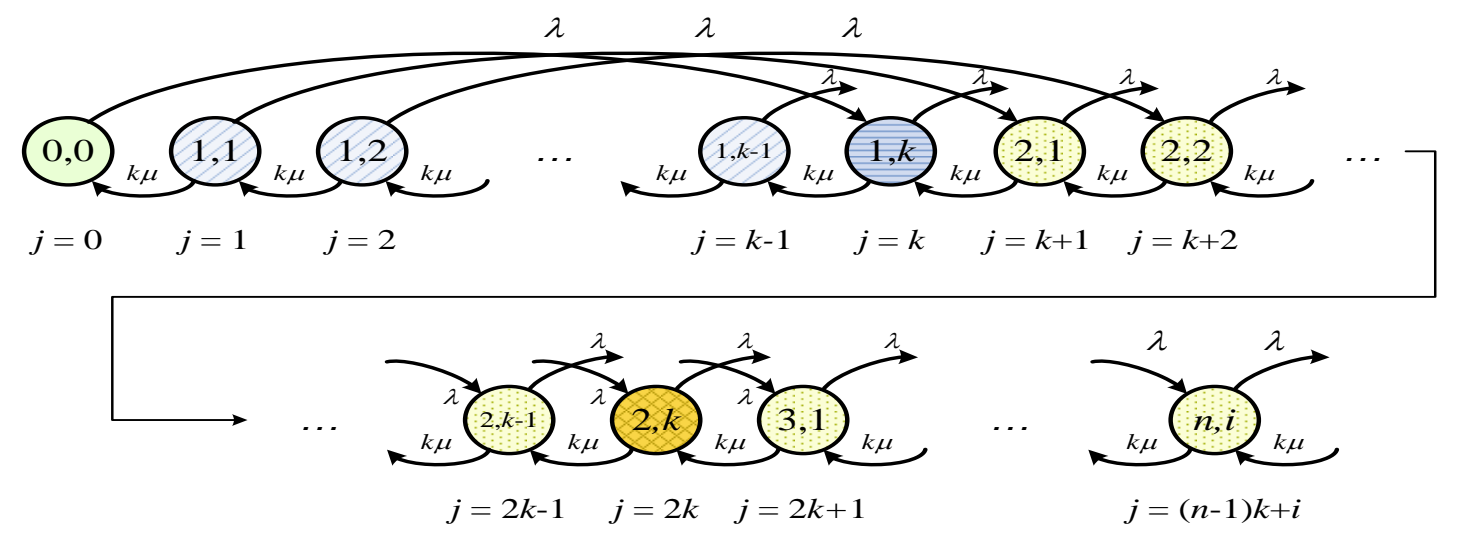

$p_{n, i}(t+\Delta t)=p_{n, i}(t)(1-\lambda \Delta t-k \mu \Delta t)+p_{n, i+1}(t)(k \mu \Delta t)+p_{n-1, i}(t)(\lambda \Delta t) \quad n \geq 2,1 \leq i \leq k$

$p_{n, k}(t+\Delta t)=p_{n, k}(t)(1-\lambda \Delta t-k \mu \Delta t)+p_{n, k+1}(t)(k \mu \Delta t)+p_{n-1, i}(t)(\lambda \Delta t) \quad n \geq 2,1 \leq i \leq k$

At steady state for $\mathrm{n}>0, \quad 1 \leq i \leq k$

$-\lambda p_{0,0}+k \mu p_{1,1}=0$

If $n=1$, then

$\begin{cases}-(\lambda+k \mu) p_{1, i}+k \mu p_{1, i+1}=0 & , 1 \leq i \leq k-1 \\ -(\lambda+k \mu) p_{1, k}+k \mu p_{2,1}+\lambda p_{0}=0 & \end{cases}$

if $n \geq 2$, then

$\left\{\begin{array}{l}-(\lambda+k \mu) p_{n, i}+k \mu p_{n, i+1}+\lambda p_{n-1, i}=0 \quad, 1 \leq i \leq k-1 \\ -(\lambda+k \mu) p_{n, k}+k \mu p_{n+1,1}+\lambda p_{n-1, k}=0\end{array}\right.$

We obtained the total phase serivce is :

$$
\begin{aligned}
G(z)=\sum_{n=1}^{\infty} \sum_{i=1}^{k} p_{n, i} z^{k(n-1)+i}+p_{0}=\frac{p_{0}(1-z)}{1-z(1+r)+r z^{k+1}} \\
W_{q}=\sum_{n=1}^{\infty} \sum_{i=1}^{k}\left[\frac{k(n-1)+i}{k \mu}\right] p_{n, i}=\left.\frac{1}{k \mu} G^{\prime}(z)\right|_{z=1} \\
=\mathrm{E}\left(N_{q}\right) \frac{1}{\mu}+\mathrm{E}(I) \frac{1}{k \mu}
\end{aligned}
$$


If we let $j=(n-1) k+i, n \geq 0,1 \leq i \leq k$ be the total number of phases for (n,i) state in the system with problem $p_{j}^{(p)} \cdot p_{j}=0, j>0, p_{n}=\sum_{i=1}^{k} p_{n, i}=\sum_{j=(n-1) k+1}^{n k} p_{j}^{(p)}$

$p_{j}^{(p)}$ : the probability of $j$ in the bulk-input system. Then we rewrite

$$
\begin{aligned}
& -\lambda p_{0}+k \mu p_{1}=0 \\
& -(\lambda+k \mu) p_{n}+k \mu p_{n+1}+\lambda p_{n-k}=0 \quad, n \geq 1
\end{aligned}
$$

The Erlangian type $k$ service model is equivalent to Bulk input model where $c_{k}=1, c_{x}=0, x \neq k$.

$$
p_{0}=1-\frac{\lambda \mathrm{E}(X)}{k \mu}=1-\frac{k \lambda}{k \mu}=1-\frac{\lambda}{\mu}=1-\rho
$$

Using partial fraction expansion may yield

$$
G(z)=(1-\rho) \sum_{i=1}^{k} \frac{A_{i}}{\left(1-\frac{z}{z_{i}}\right)} \quad \text { where } \quad A_{i}=\prod_{\substack{n=1 \\ n \neq i}}^{k} \frac{1}{\left(1-\frac{z_{i}}{z_{n}}\right)}
$$

And then

$$
p_{j}^{(P)}=(1-\rho) \sum_{i=1}^{k} A_{i}\left(z_{i}\right)^{-j} \quad ; \quad p_{n}=\sum_{j=(n-1) k+1}^{n-k} P_{j}^{(P)}
$$

The performance measures

$$
\begin{aligned}
W_{q} & =\mathrm{E}\left(T_{q}\right)=\mathrm{E}\left(N_{q}\right) \frac{1}{\mu}+\mathrm{E}(I) \frac{1}{k \mu} \\
& =\sum_{n=1}^{\infty} \sum_{i=1}^{k}\left[\frac{k(n-1)+i}{k \mu}\right] p_{n, i}+0 \cdot p_{0} \\
& =\left.\frac{1}{k \mu} \mathrm{G}^{\prime}(\mathrm{z})\right|_{\mathrm{z}=1} \\
\left.G^{\prime}(z)\right|_{z=1}=\frac{(k+1) \rho}{2(1-\rho)}, \quad & W_{q}=\frac{(k+1) \rho}{2 k \mu(1-\rho)}=\frac{k+1}{2 k} \cdot \frac{\lambda}{\mu(\mu-\lambda)} \\
L_{q}=\lambda W_{q}=\frac{k+1}{2 k} \cdot \frac{\lambda(\lambda)}{\mu(\mu-\lambda)}, & W=W_{q}+\frac{1}{\mu}=\frac{k+1}{2 k} \cdot \frac{\lambda}{\mu(\mu-\lambda)}+\frac{1}{\mu}
\end{aligned}
$$




$$
L=\lambda W=\frac{k+1}{2 k} \cdot \frac{\lambda^{2}}{\mu(\mu-\lambda)}+\frac{\lambda}{\mu}
$$

$G^{\prime}(z)=\frac{(k+1) \lambda}{2(\mu-\lambda)}$ denotes the average total phase in the system.

\section{Fuzzy Queues with k-phase infinite capacity:}

Consider a queueing system in k-phases single server facility, general customers discipline, and the size of source population is infinite, in which arrivals occur as Poisson process with at a fuzzy rate $\bar{\lambda}$, and all the service patterns are exponential distribution with fuzzy rate $\bar{\mu}$. In this model of arrival and service rates are defined as $\bar{\lambda}=\left\{\left(x, \mu_{\bar{\lambda}}(x)\right) / x \in X\right\}$ and $\bar{\mu}=\left\{\left(y, \mu_{\bar{\lambda}}(y)\right) / y \in Y\right\}$ where $\mathrm{X}$ and Y are the crisp rate, and $\mu_{\bar{\lambda}}(x)$ and $\mu_{\bar{\mu}}(y)$ are the corresponding membership functions. The performance of the system is mentioned by $\mathrm{P}(\mathrm{x}, \mathrm{y})$. If $\bar{\lambda}$ and $\bar{\mu}$ are fuzzy numbers then $\mathrm{P}(\bar{\lambda}, \bar{\mu})$ is also fuzzy number. On the basis of Zadeh's extension principle [23]. The performance measure functions is defined as $\mu_{\bar{p}(\bar{\lambda}, \bar{\mu})}(z)=\sup \min \left\{\mu_{\bar{\lambda}}(x), \mu_{\bar{\mu}}(y) / z=p(x, y)\right\}$.- (i) Without loss of generality, assume that the performance measure of interest is $L_{q}$. From the knowledge of traditional queueing teory expected system length and waiting queue length for a crisp queueing system with infinite capacity is $w_{q} \lambda=L_{q}$ and $w_{q}=\frac{1+(1 / k)}{2} * \frac{\rho}{(1-\rho) \mu}$ where $\rho$ is called the traffic intensity. From $(*)$, the memberhip function of $\overline{W_{q}}$ is

$$
\begin{aligned}
& \mu_{\overline{w_{q}}}(z)=\sup \min \left\{\mu_{\bar{\lambda}}(x), \mu_{\bar{\mu}}(y) / z=\left(\frac{k+1}{2 k}\right) \frac{\rho}{\mu(1-\rho)}\right\}------- \text { (ii) } \\
& \mu_{\overline{L_{q}}}(z)=\sup \min \left\{\mu_{\bar{\lambda}}(x), \mu_{\mu}(y) / z=\lambda \overline{w_{q}}\right\}
\end{aligned}
$$

and

Now, the pair of nonlinear program is developed the basis extension principle and find the degree of certainity of the system performance analyze via statitical inference.

\section{Solution Procedure:}

To construct the membership function $\mu(\bar{\lambda}, \bar{\mu})$ is formation on the basis of derivation of $\alpha$ - cut of arrival and service as follows:

$\overline{\lambda_{\alpha}}=\left[x_{\alpha}^{l}, x_{\alpha}^{u}\right]=\left[\min \left\{x / \mu_{\lambda}(x) \geq \alpha\right\}, \max \left\{x / \mu_{\lambda}(x) \geq \alpha\right\}\right]$

$\overline{\mu_{\alpha}}=\left[y_{\alpha}^{l}, y_{\alpha}^{u}\right]=\left[\min \left\{x / \mu_{\mu}(y) \geq \alpha\right\}, \max \left\{y / \mu_{\mu}(y) \geq \alpha\right\}\right]$

The arrival and service rate can also be represented by different levels of confidence intervals [14,19].consequently, $\mathrm{FM} / \mathrm{FE}_{\mathrm{k}} / 1$ can be reduced to a family of crisp $\mathrm{M} / \mathrm{E}_{\mathrm{k}} / 1$ queues with different $\alpha$-level sets $\left\{\left(\lambda_{\alpha}, \mu_{\alpha}\right) / 0<\alpha \leq 1\right\}$. These two set of expressions are relationship between ordinary sets and fuzzy sets [5].By the convexity of a fuzzy number bounds of these interval function of $\alpha$ can be obtained 
as $x_{\alpha}^{l}=\min \left(\mu_{\lambda}^{-1}(\alpha)\right), x_{\alpha}^{u}=\max \left(\mu_{\lambda}^{-1}(\alpha)\right), y_{\alpha}^{l}=\min \left(\mu_{\mu}^{-1}(\alpha)\right)$ and $y_{\alpha}^{u}=\max \left(\mu_{\mu}^{-1}(\alpha)\right)$. Clearly, as defined in[3], the membership function of $(\bar{\lambda}, \bar{\mu})$ is also parameterized by $\alpha$. Consequently, we can use its $\alpha$-cut to construct its membership function. According to (i),$\mu_{\overline{L_{q}}}(z)$ and $\mu_{\overline{w_{q}}}(z)$ is the minimum o $\mu_{\bar{\lambda}}(x)$ and $\mu_{-}(y)$. We need to either $\mu_{\bar{\lambda}}(x)=\alpha$ and $\mu_{-}(y) \geq \alpha$ or $\mu_{\bar{\lambda}}(x) \geq \alpha$ and $\mu_{-}(y)=\alpha$ such that $L_{q}(z)=\lambda W_{q}$ and $W_{q}=\frac{1+1 / k}{2}\left(\frac{\rho}{\mu(1-\rho)}\right)$ to satisfy that $\mu_{L_{q}}(z)=\alpha$ and $\mu_{W_{q}}(z)=\alpha$. According the definition of (i), $y \in \mu(\alpha)$ and $x \in \lambda(\alpha)$ can be replaced by $x \in\left[x_{\alpha}^{l}, x_{\alpha}^{u}\right]$ and $y \in\left[y_{\alpha}^{l}, y_{\alpha}^{u}\right]$ respectively, Thus, based on (ii \&iii) to find the membership function of $\mu_{L_{q}}(z)$ and $\mu_{W_{q}}(z)$, it suffies to find the lower and upper bound $\left[L_{q_{\alpha}}^{l}, L_{q_{\alpha}}^{u}\right]$ and $\left[W_{q_{\alpha}}^{l}, W_{q_{\alpha}}^{u}\right\rfloor$ of the $\alpha$-cuts of $\mu_{L_{q}}(z)$ and $\mu_{W_{q}}(z)$, which can be rewritten as: $L_{q \bar{\alpha}}^{l}(z)=\min \left\{\left(\frac{k+1}{2 k}\right) \frac{\rho^{2}}{(1-\rho)}\right\} \quad$-----(iv) $\quad$ and $L_{q-\alpha}^{u}(z)=\max \left\{\left(\frac{k+1}{2 k}\right) \frac{\rho^{2}}{(1-\rho)}\right\}$---- (v) s.t $x_{\alpha}^{l} \leq x \leq x_{\alpha}^{u}$ and $y_{\alpha}^{l} \leq y \leq y_{\alpha}^{u}$. There are several effective and efficient methods for solving these problems[20]. Moreover, analyze how to change the optimal solution as $x_{\alpha}^{l}, x_{\alpha}^{u}, y_{\alpha}^{l}$ and $y_{\alpha}^{u}$ where $\alpha \in[0,1]$; they fall into the category of NLP[21]. If $L_{q_{\alpha}}=\left[L_{q_{\alpha}}^{l}, L_{q_{\alpha}}^{u}\right\rfloor$ and $W_{q_{\alpha}}=\left\lfloor w_{q_{\alpha}}^{l}, w_{q_{\alpha}}{ }^{u}\right\rfloor$ are invertible with respect to $\alpha$, then the left and right shape of the function is $[\mathrm{L}(\mathrm{z}), \mathrm{R}(\mathrm{z})]=L^{-1} q_{\alpha}$ and $[\mathrm{L}(\mathrm{z}), \mathrm{R}(\mathrm{z})]=W_{q_{\alpha}}^{-1}$ can be obtained from membership function $\mu_{W_{q}}(z)$ and $\mu_{L_{q}}(z)$ constructed:

$$
\mu_{\mathrm{L}_{\mathrm{q}}}(z) \& \mu_{\mathrm{W}_{\mathrm{q}}}(z)=\left\{\begin{array}{ll}
L(z) & z_{1} \leq z \leq z_{2} \\
1 & z=z_{2} \\
R(z) & z_{2} \leq z \leq z_{3}
\end{array}\right\}
$$

Otherwise, if the values of $L_{q_{\alpha}}$ and $W_{q_{\alpha}}$ cannot obtained analytically, the numerical solutions for $L_{q_{\alpha}}=\left[L_{q_{\alpha}}{ }^{l}, L_{q_{\alpha}}{ }^{u}\right]$ and $W_{q_{\alpha}}=\left[w_{q_{\alpha}}{ }^{l}, w_{q_{\alpha}}{ }^{u}\right]$ at different possibility level of $\alpha$ can be collected to approximate the shapes of $\mathrm{L}(\mathrm{z})$ and $\mathrm{R}(\mathrm{z})$. That is, the set of interval is reveals the shape of $\mu_{\mathrm{L}_{\mathrm{q}}}(z) \& \mu_{\mathrm{W}_{\mathrm{q}}}(z)$ although the exact function is not known explicitly. Other membership function of performance measures can be derived in the similar manner. The fuzziness values are converted to crisp value using Roubst Ranking Technique. However, in practical point of view the management would expect without blocking the fuzzy arrival due to behaviour of the service. If the system providing worst service then the level of queue size increased. Generally any management like to avoid this kind of behaviour, in this connection we estimated the queues parameters used in statistical inference. The statistical manner can be derived in section.

\section{Estimation parameters of an FM/FEk/1 Queue:}

Consider the estimating parameters of a homogeneous Markov chain, such as a simple birth-death process with constant birth rate $\lambda$ and constant death rate $\mu$. This corresponds to an M/Ek/1 queue with an arrival rate $\lambda$ and service rate $\mu$. To estimate the arrival rate $\lambda$, we observe that the arrival process is 
Poisson. If the time required to observe " $\mathrm{n}$ " arrival denoted by $\varphi(n)$ then the maximum likelihood estimator of $\bar{\lambda}$ is $\bar{\lambda}=\frac{n}{s_{n}}$. Also, sine $2 \bar{\lambda} \varphi(n)$ has a chi-square distribution with 2 degrees of freedom, a $100(1-\alpha) \%$ confidence interval for $\bar{\lambda}$ is given by $\left(\frac{\chi_{2 n ; 1-\alpha / 2}^{2}}{2 s_{n}}, \frac{\chi_{2 n ; \alpha / 2}^{2}}{2 s_{n}}\right)$. In order to estimate the service rate $\mu$, our assumptions of the service times are independent exponentially distributed random variables. Thus, if the service times $X_{1}, X_{2}, X_{3} \ldots \ldots \ldots . . . . X m$ have been observed, and if we get $\phi(m)=\sum_{i=1}^{m} X_{i}$ the maximum likelihood estimator of $\mu$ is given by $\bar{\kappa}=\frac{m}{\phi(m)}$ where $\phi(m)$ may also be interpreted as the total busy time of the server during the observation period. Noting that $2 \bar{\mu} \phi(m)$ has $\chi_{2 m}^{2}$ distribution, we get a $100(1-\alpha) \%$ confidence interval for $\bar{\mu}$ as $\left(\frac{\chi_{2 m ; 1-\alpha / 2}^{2}}{2 y_{m}}, \frac{\chi_{2 m ; \alpha / 2}^{2}}{2 y_{m}}\right)$. Server Utilization $\rho$ is now estimated by $\bar{R}=\frac{\varphi(m) / m}{\phi(n) / n}$. Now, since $\phi(m)$ and $\varphi(n)$ are independent, and $2 \bar{\mu} \phi(m)$ and $2 \bar{\lambda} \varphi(n)$ are both chi-square distributed, it follows that $\bar{R} / \rho$ has an "F" distribution with $2 \mathrm{~m}$ and 2 n degrees of freedom. To obtain a 100(1- $\alpha) \%$ confidence interval for $\rho$.

\section{Example:}

Consider a centralize parallel processing system in which the arrival at different level of phases. The phase size random variable $\mathrm{K}$ is a Erlang distribution, which is often studied in crisp bulk arrival with the expected value of $\mathrm{k}=3$; i.e., the arrival of the system in accordance with a Poisson Process, and the service times follow an Erlang distribution. Both the group arrival and service rate are triangular fuzzy numbers represented by $\lambda=[1,5,7]$ and $\mu=[9,10,11]$ per minute, respectively. The system manager wants to evaluate the performance measures of the system such as the expected number of customers in the queue and waiting in the queue and to analyze optimality level of the system.

It is clear that in this example the steady-state condition $\rho=\frac{3 x}{y}<1$ is satisfied, thus the performance measures of interest can be constructed by using the approach stated in solution procedure following (iv $\& v$ ), two pairs of MINLP models for deriving the membership function of $\overline{L_{q}}$ can be formulated, whose solutions are as follows:

$$
{ }^{L} L_{q}(\alpha)=\frac{32 \alpha^{2}+16 \alpha+2}{15 \alpha^{2}-195 \alpha+330} \quad \text { and } \quad{ }^{U} L_{q}(\alpha)=\frac{8 \alpha^{2}-56 \alpha+98}{9 \alpha^{2}+87 \alpha+54}
$$

The inverse functions of ${ }^{U} L_{q}(\alpha)$ and ${ }^{L} L_{q}(\alpha)$ exist, which give the membership function $\mu_{\bar{L}_{q}}(Z)$ as 


$$
\mu_{\bar{L}_{q}}(Z)=\left\{\begin{array}{cc}
\frac{(195 z+16) \pm \sqrt{18225 z^{2}+48600 z}}{30 z-64} & \text { for } \frac{1}{165} \leq z \leq \frac{1}{3} \\
1 & \text { for } \frac{1}{3} \leq z \leq \frac{49}{27} \\
\frac{-(87 z+56) \pm \sqrt{5625 z^{2}+15000 z}}{18 z-16} & \text { for } \frac{49}{27} \leq z \leq 3
\end{array}\right.
$$

Similarly we derived,

$$
{ }^{L} w_{q}(\alpha)=\frac{8 \alpha+2}{15 \alpha^{2}-195 \alpha+330} \quad \text { and } \quad{ }^{U} w_{q}(\alpha)=\frac{14-4 \alpha}{9 \alpha^{2}+87 \alpha+54}
$$

The Inverse function of is ${ }^{L} w_{q}(\alpha)$ and ${ }^{U} w_{q}(\alpha)$ exist, which give the membership function

$$
\mu_{w_{q}}(Z)=\left\{\begin{array}{cl}
\frac{(195 z+8) \pm \sqrt{18225 z^{2}+3240 z+64}}{30 z} & \text { for } \frac{1}{165} \leq z \leq \frac{13}{150} \\
1 & \text { for } \frac{13}{150} \leq z \leq \frac{1}{15} \\
\frac{-(87 z+4) \pm \sqrt{5625 z^{2}+1200 z+16}}{18 z} & \text { for } \frac{1}{15} \leq z \leq \frac{7}{27}
\end{array}\right.
$$

Table 1: $\quad$ The $\alpha$ cuts of the performance measures at $11 \alpha$ values are as follows;

\begin{tabular}{|c|c|c|c|c|c|c|c|c|c|c|c|c|}
\hline $\mathrm{A}$ & $l_{x}^{(\alpha)}$ & $u_{x}^{(\alpha)}$ & $l_{y}^{(\alpha)}$ & $u_{y}^{(\alpha)}$ & ${ }^{L} L_{q}(\alpha)$ & ${ }^{U} L_{q}(\alpha)$ & ${ }^{L} w_{q}(\alpha)$ & ${ }^{U} w_{q}(\alpha)$ & $\operatorname{Core}\left(L_{q}^{\alpha}\right)$ & $\operatorname{Core}\left(W_{q}^{\alpha}\right)$ & $L_{q}^{\alpha} \%$ & $W_{q}^{\alpha} \%$ \\
\hline 0 & 1 & 7 & 9 & 11 & 0.0061 & 1.8148 & 0.0061 & 0.2593 & 0.1327 & 0.91945 & 91 & 13 \\
\hline 0.1 & 1.4 & 6.8 & 9.1 & 10.9 & 0.0126 & 1.4728 & 0.0091 & 0.2166 & 0.11285 & 0.7427 & 74 & 11 \\
\hline 0.2 & 1.8 & 6.6 & 9.2 & 10.8 & 0.0222 & 1.2140 & 0.0123 & 0.1839 & 0.0981 & 0.6181 & 61 & 9 \\
\hline 0.3 & 2.2 & 6.4 & 9.3 & 10.7 & 0.0355 & 1.0125 & 0.0161 & 0.1582 & 0.0871 & 0.524 & 52 & 8 \\
\hline 0.4 & 2.6 & 6.2 & 9.4 & 10.6 & 0.0531 & 0.8520 & 0.0204 & 0.1374 & 0.0789 & 0.45255 & 45 & 7 \\
\hline 0.5 & 3 & 6 & 9.5 & 10.5 & 0.0762 & 0.7218 & 0.0254 & 0.1203 & 0.07285 & 0.399 & 39 & 7 \\
\hline 0.6 & 3.4 & 5.8 & 9.6 & 10.4 & 0.1059 & 0.6148 & 0.0311 & 0.1060 & 0.06855 & 0.3603 & 36 & 6 \\
\hline 0.7 & 3.8 & 5.6 & 9.7 & 10.3 & 0.1438 & 0.5254 & 0.0378 & 0.0939 & 0.06585 & 0.3346 & 33 & 6 \\
\hline 0.8 & 4.2 & 5.4 & 9.8 & 10.2 & 0.1922 & 0.4508 & 0.0458 & 0.0835 & 0.06465 & 0.3215 & 32 & 6 \\
\hline 0.9 & 4.6 & 5.2 & 9.9 & 10.1 & 0.2539 & 0.3874 & 0.0552 & 0.0745 & 0.06485 & 0.32065 & 32 & 6 \\
\hline 1 & 5 & 5 & 10 & 10 & 0.3333 & 0.3333 & 0.0667 & 0.0667 & 0.0667 & 0.3333 & 33 & 6 \\
\hline
\end{tabular}

Using (sec 4) we analyze system performance optimality (XL-Stat 2016) relation between the average number of customer waiting in the queue and customer has to spend the time in queue. 


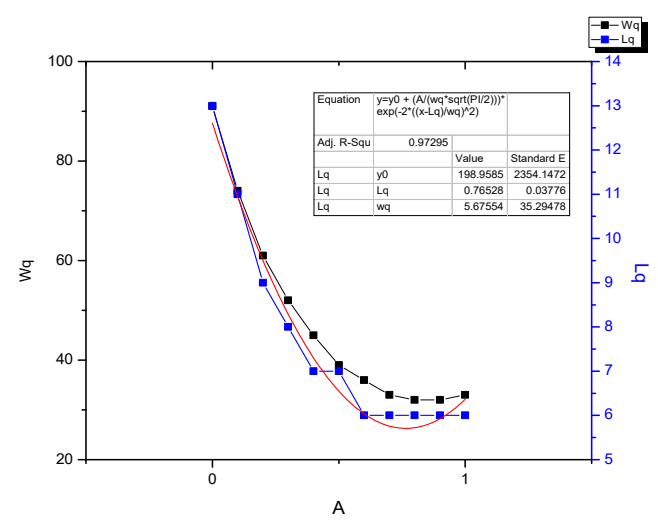

(Fig (i))

Table 2: Status of Lq \& Wq in Reduced chi-sq

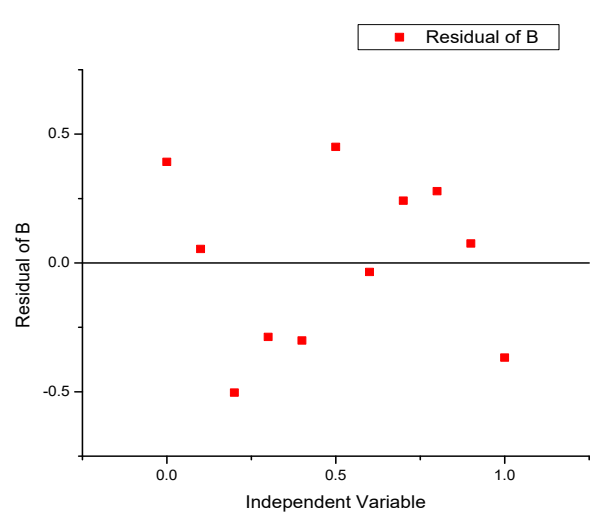

(Fig (ii))

Table 3: $\quad$ Solution of the Optimality in eleven

Samples;

\begin{tabular}{|l|l|}
\hline D.F & 7 \\
\hline Adj.R.Square & 0.97295 \\
\hline Red.Chi.Sqr & 0.15198 \\
\hline Res.Sum of Sqr & 1.06386 \\
\hline
\end{tabular}

Table 4: $\quad$ Regular Residual of the $\mathrm{Lq}$ and $\mathrm{Wq}$

\begin{tabular}{|c|l|l|l|}
\hline \multicolumn{2}{|c|}{ Lq } & \multicolumn{2}{c|}{ Wq } \\
\hline Value & Error & Value & Error \\
\hline 0.76528 & 0.03776 & 5.6753 & 5.2947 \\
\hline
\end{tabular}

\begin{tabular}{|c|c|c|c|}
\hline $\begin{array}{c}\text { Degree of } \\
\text { Uncertainity }\end{array}$ & $\begin{array}{c}\text { Regular Residuals } \\
\text { of } W q\end{array}$ & $\begin{array}{c}\text { Regular } \\
\text { Residuals } L q\end{array}$ & Gauss Fit of $W q$ \\
\hline 0 & 0.39179 & 0.39179 & 12.60821 \\
\hline 0.1 & 0.05436 & 0.05436 & 12.43043 \\
\hline 0.2 & -0.50364 & -0.50364 & 12.25484 \\
\hline 0.3 & -0.2875 & -0.2875 & 12.08146 \\
\hline 0.4 & -0.3017 & -0.3017 & 11.91028 \\
\hline 0.5 & 0.45012 & 0.45012 & 11.74132 \\
\hline 0.6 & -0.03483 & -0.03483 & 11.57458 \\
\hline 0.7 & 0.24154 & 0.24154 & 11.41007 \\
\hline 0.8 & 0.27819 & 0.27819 & 11.24779 \\
\hline 0.9 & 0.075 & 0.075 & 11.08775 \\
\hline 1 & -0.36728 & -0.36728 & 10.92996 \\
\hline
\end{tabular}

\section{Conclusion:}

In this study, we considered an optimal K-Policy in the $\mathrm{FM} / \mathrm{FE}^{(\mathrm{k})} / 1$ queue with general server setup time. Especially, the entire queueing system performances are based in the server providing the service status. Because, the level of system performance is optimized in service status only. The arrival and service rate are imprecise by applying the $\alpha$-cuts. The idea is based on Zadeh's extension principle transform to the fuzzy queue that can be described a pair of MINLP models. By applying the $\alpha$-cut approach, the performance measure at different level can be derived. Consider the average length $\overline{L_{q}}$ and 
the customer waiting queue length $\overline{W_{q}}$. If, the degree of certainty $\alpha=0$ then $\overline{L_{q}}=\left[\begin{array}{ll}0.0061 & 1.8148\end{array}\right]$ and $\overline{W_{q}}=[0.0061,0.2593]$. If, the degree of certainty $\alpha=1$ then $\overline{L_{q}}$ is $0.3333 \& \overline{W_{q}}=0.0667$. The fuzziness values are converted to crisp value using Roubst ranking function the value of $\overline{W_{q}} \& \overline{L_{q}}$ is $0.1327 \& \quad 0.91945$. The degree of uncertainity is analyzed the chi square distribution $\alpha=[0.4$ to $0.6]$ in this range performance of the system is not optimized. After that we analyze which customer has to suffer in service time using the statistical interference. Using our proposed methodology reduced chi square distribution in the degrees of freedom seven and to the optimum level of system performance is 0.1518. Suppoe we analyze entire system the error value of $\overline{W_{q}} \& \overline{L_{q}}$ is $0.03776 \& 5.2947$ and to adjusted the chi square value is 0.97295 . Our porposed methodology is most helpful for the system operation (simulation) studies and then we quickly indentified the blocking to the system.

\section{References:}

[1] Barak,S., Fallahnezhad,M.S (2012). Cost Analysis of Fuzzy Queuing Systems International Journal of Applied Operational Research Vol. 2(2), pp. 25-36.

[2] Buckley, J. J., Feuring, T., Hayashi, Y., (2001). Fuzzy queuing theory revisited. International Journal of Uncertainty, Fuzziness and Knowledge-Based Systems, 9 (5), pp 527-537.

[3] Buckley.J.J .,(1990). Elementaty queueing theory based on possibility theory, Fuzzy Set Syst, Vol(37), pp 43-52.

[4] Chanas.S, Nowakowski.M.,(1988). Single value simulation on Fuzzy variable, Fuzzy Set Syst, Vol(21), pp 43-57.

[5] Chen, S. P., (2006). A bulk arrival queuing model with fuzzy parameters and varying batch sizes. Applied Mathematical Modeling, 30, 920-929.

[6] De Cooman, G., (2005). A behavioral model for vague probability assessments. Fuzzy Sets and Systems, 154, 305-358.

[7] Jo.J.B, Tsujimura.Y, Gen.M and Yamazaki.G.,(1996). Performance evaluation of networks based on Fuzzy Queueing System. Jpn.J.Fuzzy Theor.Syst, Vol.08, pp 393-408.

[8] Kaufmann,A.,(1975). Introduction to the theory of fuzzy subsets, vol.1,Academic Press.New York,

[9] Kao, C., Li, C., Chen, S., (1999). Parametric programming to the analysis of fuzzy queues. Fuzzy Sets and Systems, 107, 93-100.

[10] Ke,J.C., (2004). Bi-level control for batch arrival queues wit an early setup an un-reliable server. Appl.Mat.Moell. Vol.28, pp 469-485.

[11] Li, R. J., Lee, E. S., (1989). Analysis of fuzzy queues. Computers and Mathematics with Applications, 17(7), pp 1143-1147.

[12] Liou, T. S., Wang, M. J. J., (1992). Ranking fuzzy numbers with integral value. Fuzzy Sets and Systems, 50, 247-255.

[13] Nakamura, K., (1986). Preference relations on a set of fuzzy utilities as a basis for decision making. Fuzzy Sets and Systems, 20, 147-162.

[14] Negi,D.S., Lee,E.S (1988). Analysis and simulation of fuzzy queue. Fuzzy Sets Syst. Pp 214357.

[15] Palpandi,B.,Geetharamani,G.,(2013). Evaluation of Performance Measures of Bulk Arrival Queue WithFuzzy Parameters Using Robust Ranking Technique. International Journal of Computational Engineering Research, Vol, 03(10), pp 55-57.

[16] Prade.H.M.,(1980). An outline of Fuzzy or possibilistic model for queueing systems, in: Wang.P.P, Chang.S.K(Eds.). Fuzzy Sets, Plenum Press, New York, pp 147- 153. 
[17] Ritha,W.,Sreelekha,M.,(2011). Fuzzy N Policy Queues with Infinite CapacityJournal of Physical Sciences, Vol. 15, pp 73-82.

[18] Satish Verma.,(2015). Chi Square Method for Testing the Inter-Arrival and Service Patterns. International Journal of Mechanical Engineering, Vol.02(05), pp76-83.

[19] Shih-Pin Chen.,(2006). A bulk arrival queueing model with fuzzy parameters and varying batch sizes. Applied Mathematical Modelling, Vol.30, pp 920-929

[20] Shih-Pin Chen.,(2004). Parametric nonlinear programming for analyzing fuzzy queues with finite capacity. European Journal of Operation Research, Vol.157, pp 429-438.

[21] Sun Hur, Seung-Jin Paik.,(1999). The effect of different arrival rate on the N-policy of M/G/1 with server setup,. Applied Mathematical Modelling, Vol. 23(4), pp. 255-343

[22] ZimmermannH.J.,(2001). Fuzzy Set theory and its Application, fourth ed., Kluwer Acaemic, Boston.

[23] Zadeh.L.A.,(1978). Fuzzy sets as a basis for a theory of possibility. Fuzzy Sets Syst, Vol.01, pp 03-28. 